\author{
Juraj Miček, Ondrej Karpiš, Veronika Olešnaníková \\ University of Zilina \\ Univerzitna 8215/1 \\ 01026 Zilina, Slovakia
}

Email: \{Juraj.Micek, Ondrej.Karpis, Veronika.Olesnanikova\}@fri.uniza.sk

\title{
Mobile sensor elements based on robotic platform YROBOT
}

\begin{abstract}
During the years 2014-2015 a kit called Yrobot was developed at the Department of technical cybernetics. The kit is a mobile robotic platform designed mainly to support technical education at high schools and universities. Since the kit was presented at conferences RAAD 2014 and EDERC 2014 for the first time, several expansion modules and interesting applications were developed. One application is presented in this paper. The application uses the mobility of wireless sensors to map the chosen area. As an example, we realized RSSI measurements and visualized them as a function of sensor position. Obviously, one can find many similar tasks: mapping of temperature or gas concentration in given space and the like. Our ultimate goal is to analyze the possibilities of using RSSI measurements for indoor localization. However, at this stage of research, we were focused just on acquiring the data and their subsequent visualization.
\end{abstract}

\section{InTRODUCTION}

$I^{\mathrm{s}}$ N PREVIOUS years, simple mobile system Yrobot was developed at the Department of technical cybernetics. This system was designed especially for teaching IT subjects in the high schools. The concept of the system, its features and functions, as well as first experience of deployment of the system in teaching, were presented at the conference RAAD in 2014 [2, 3]. Original author's intent was as following: "By using a simple technical device to increase the motivation of high school students in studying technical fields, particularly in information technologies." Thanks to the long-term support of the Volkswagen Slovakia Foundation, Yrobot kits were delivered to the high schools. Our team prepared detailed textbooks also [1]. Based on the Yrobot platform, competition for high school students "Program the robot - Yrobot Cup" was organized and other supporting activities were carried out. Note that the robotic platform has the nature of open source hardware and thus, we suppose the development of other applications (hardware and software modules) directly by high schools and universities students. In addition to the typical educational mission, the platform allows verification of various methods of information processing. The data can be obtained from the real environment using different sensor modules.

Many modules were developed to sense:

- temperature and humidity,

- parameters of magnetic fields,

- acceleration,

- illumination,
- sound ( $20 \mathrm{~Hz}$ to $4 \mathrm{kHz}$ ),

- concentrations of $\mathrm{CO} 2, \mathrm{CO}, \mathrm{NOx}$,

- distance (using ultrasound),

- optical reflectivity of the surface.

Connection of sensors with mobile platform brings many solutions that often go beyond the simple application tasks.

The mobile platform is formed by a simple MCU ATmega 16, a pair of DC motors and supporting electronics. The block structure of the platform is shown in Fig.1.

Wireless connectivity of mobile robots can further expand set of tasks that can be successfully solved by Yrobot platform. Therefore, we developed a communication module providing wireless communication between the mobile platforms.

\section{RF COMmunication Modules}

We assume that wireless connectivity of mobile robots extend significantly the application potential of the platform. Recall that Yrobot was originally developed as an autonomous device capable of solving simple tasks based on the status of its sensors (line following, obstacles avoiding, area browsing). Wireless communication changes the autonomous Yrobot platform to the cooperative system of multiple robots for solving problems using data fusion. Providing connectivity allows transition from autonomous to multirobotic system and the robust solutions of complex challenges. To allow effective communication between elements of the system a variety of communication technologies (protocols and network topologies) can be used. First, we have decided to implement three separate communication modules operating in the ISM $2.4 \mathrm{GHz}$ band.

In table I. are shown main parameters of selected communication technologies.

All developed modules are connected via connectors of the motherboard to the microcontroller on Yrobot. The motherboard provides power supply to the communication modules. Communication with modules is serial, either asynchronous (UART) or synchronous (SPI). The module contains circuitry to ensure power supply, logic signals level shift and selection of communication line UART/SPI. LEDs are used to signalize status of communication module. The buttons on the module allow restarting of the module, firmware change or change of the operation mode. 


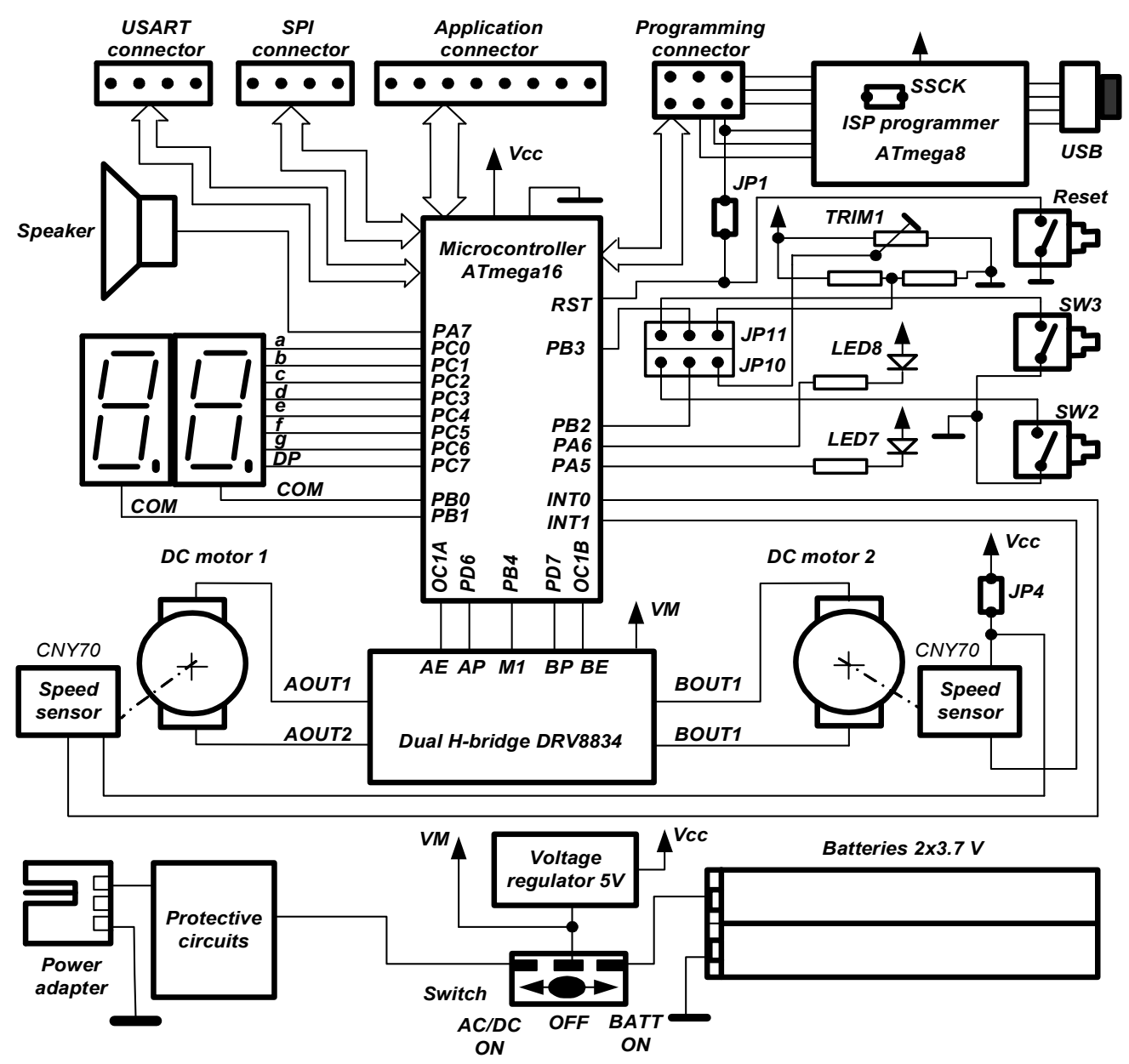

Fig 1. Block structure of Yrobot mobile platform

III. Communication Module Y-WiFi

As a first module, we developed communication module Y-WiFi. The essential element of this system is WizFi250 communication module. The WizFi250 module has an integrated LNA, printed antenna, and connector for external antenna. It offers secure communication based on protocols WEP, WPA, and WPA2. It is controlled by a set of AT commands.

Module Y-WiFi includes all other components that allow easy connection to the mobile Yrobot platform or to a personal computer. The module Y-WiFi can be connected to the PC via the USB port. FT323RL circuit was used to convert
UART to USB. Block diagram of the module is shown in Fig. 2.

The module contains two buttons $\mathrm{S} 1$ and $\mathrm{S} 2$ that allow selection of basic operation mode and restarting of the module. Status of the WizFi250 module is displayed using two LEDs - LED1 is on when the connection is established and LED 2 is on in data mode. Status of the FT323RL circuit is likewise indicated by LED3 (pulsing when data are transmitted) and LED4 (pulsing when data are received). LED5 is used to indicate the power-supply.

Jumper JMP1 allows selection between application or BOOT mode in which can be updated module firmware.

TABLE I.

Communication technologies

\begin{tabular}{|l|c|c|c|c|}
\hline Network technology & Standard & Frequency band & Embedded module & Max bit rate \\
\hline WiFi & $802.11 \mathrm{~b} / \mathrm{g} / \mathrm{n}$ & $2.4 \mathrm{GHz}$ & WizFi250 & $65 \mathrm{Mbps}$ \\
\hline Zigbee & 802.15 .4 & $2.4 \mathrm{GHz}$ & JN5168M0 \\
MRF24J40 & $250 \mathrm{kbs}$ \\
\hline Bluetooth v. 2.0 EDR & 802.15 .1 & $2.4 \mathrm{GHz}$ & BTM182 & $3 \mathrm{Mbps}$ \\
\hline
\end{tabular}




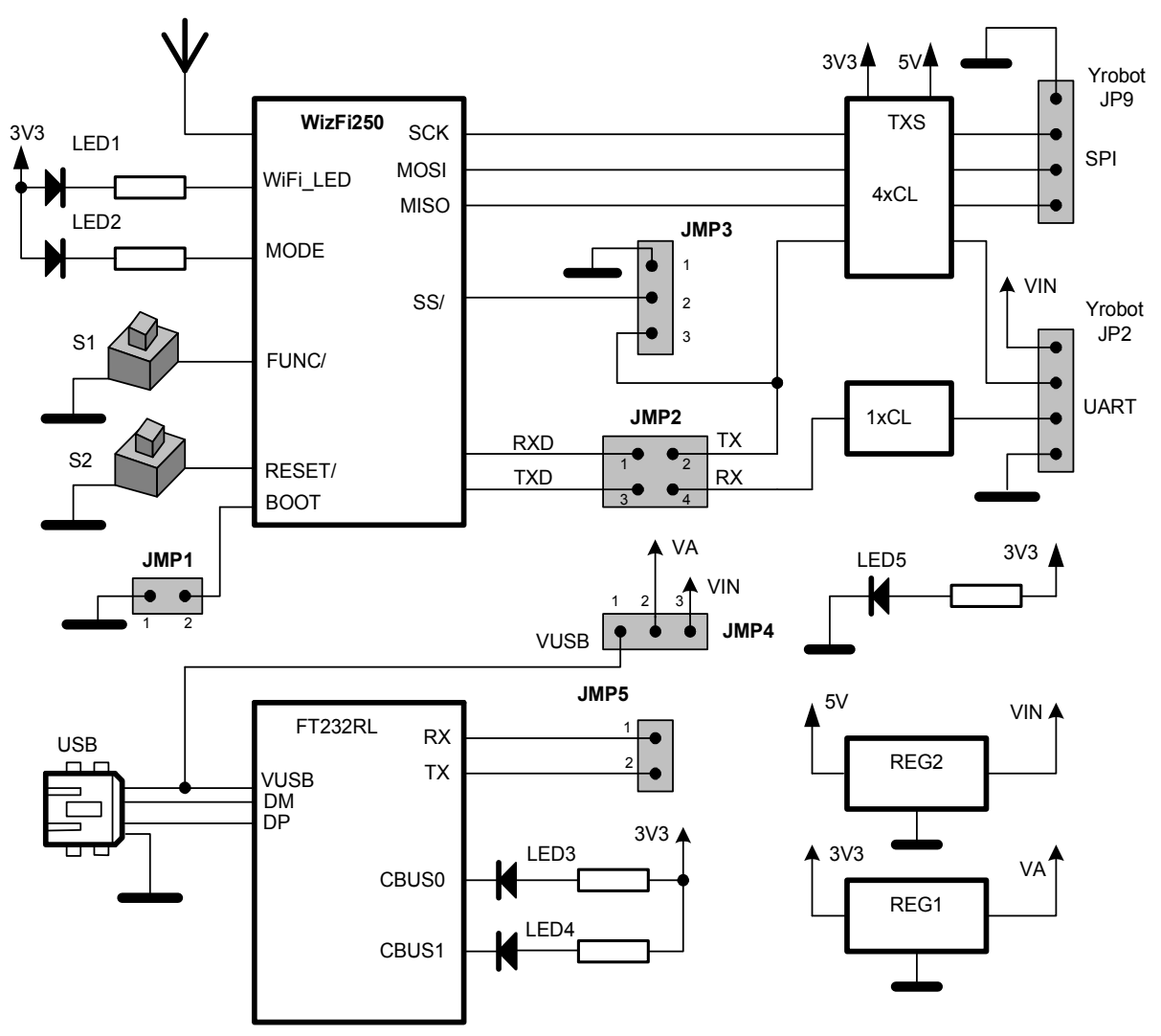

Fig 2. Block structure of Yrobot mobile platform

Jumpers JMP2 and JMP5 select connection of communication module WizFi250 to a PC (via USB) or to the Yrobot platform. It is also possible to connect a PC to the Yrobot. Jumper JMP3 is used when communicating with the module using SPI interface. Finally, with a jumper JMP4 we choose source of power supply for the module - PC USB or Yrobot batteries.

\section{EXPERIMENT}

Using the described technical means, we conducted an experiment to determine the signal strength map in the 2.4 $\mathrm{GHz}$ band. The experiment was carried out in the hallway of our workplace. WiFi internet connection in the hallway and in adjacent rooms is provided by Access Point Planet WAP4000 with the following parameters: standard 802.11g, channel 7, authentication: WPA-PSK, transmitter power set to a minimum $(-12 \mathrm{~dB})$. Transmitter power during the measurement was set to a minimum in order to magnify the signal attenuation in places distant from the AP. The measurement was carried out at the time when the hallway was empty, as the presence of people could influence the results.

Yrobot was programmed so that it can be controlled via a notebook. The Y-WiFi module was configured in Station Mode with a fixed IP address. The Y-WiFi module had an activated TCP server that listens on port 5000. Yrobot movement was based on orders received by the server. The speed of rotation of the robot's wheels was set at about 1 revolution per second. This corresponds to a speed of $0.2 \mathrm{~m} / \mathrm{s}$. Af- ter each half turn of the wheel (e.g. every $0.1 \mathrm{~m}$ ), the strength of the signal (RSSI) was measured and sent to the client (notebook).

Control notebook was also connected to a WiFi network and using the Realterm application it was connected to the Y-WiFi module. Realterm application was used for the reason that it allows to send pressed buttons to the server and record the received data to a file simultaneously. Fig. 3 shows the robotic platform Yrobot with Y-WiFi module used in the experiment.

Fig. 4 depicts a plan view of the hallway and the route of the robot during the experiment. The distance of adjacent tracks was $0.3 \mathrm{~m}$. The whole experiment was divided into three parts - the two short passages and a larger central hall were measured independently. After measurements, the three sets of data were combined into one. RSSI was measured about 1900 times during the experiment. The data were interpolated using Matlab in order to obtain values with a spatial resolution of $0.1 \mathrm{~m}$. The measured values are shown in Fig. 5. The map is consistent with our assumptions that the weakest signal is in the areas that are shaded by walls.

Note that during the measurement the antenna of Y-WiFi module was only about $7 \mathrm{~cm}$ above the ground. The situation in different height may be different.

The acquired map can be partially used for determining the position of the robot based on RSSI measurements. Obviously, the estimation of the position of the robot based on a single measurement is not possible. Significantly more 


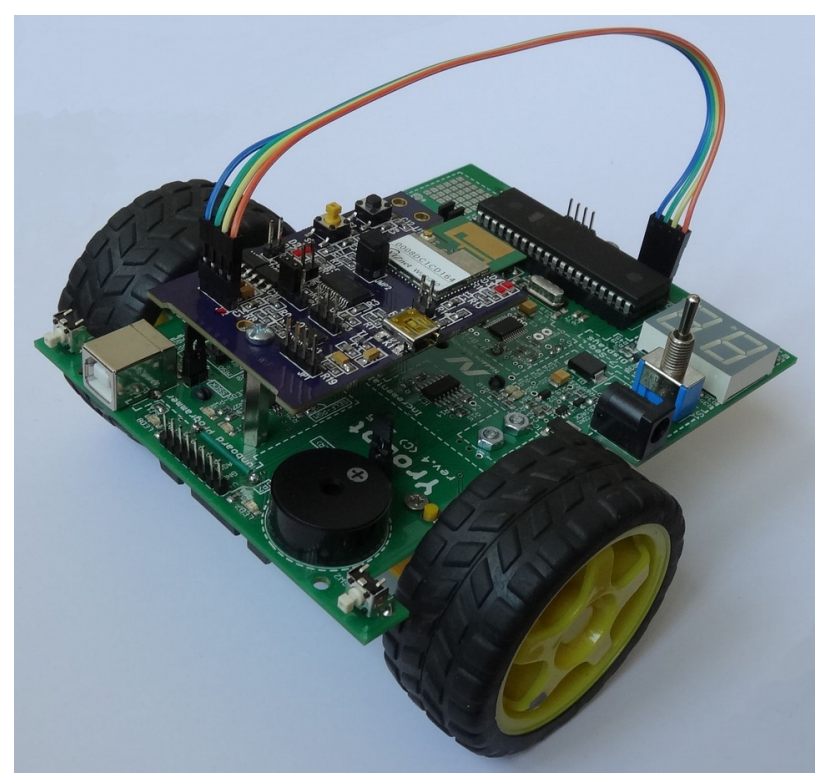

Fig 3. Mobile platform Yrobot with Y-WiFi module

precise localization should be possible with a map obtained by a directional antenna that can be rotated in different directions. Such a system is currently under development.

\section{Conclusion}

Extension of the Yrobot platform with network modules significantly expands the variety of applications that can be

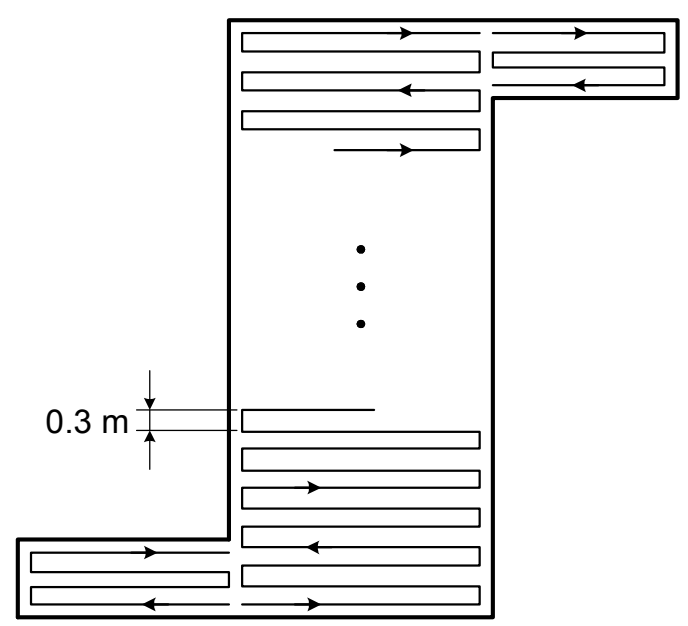

Fig 4. Plan view of the hallway and a robot route

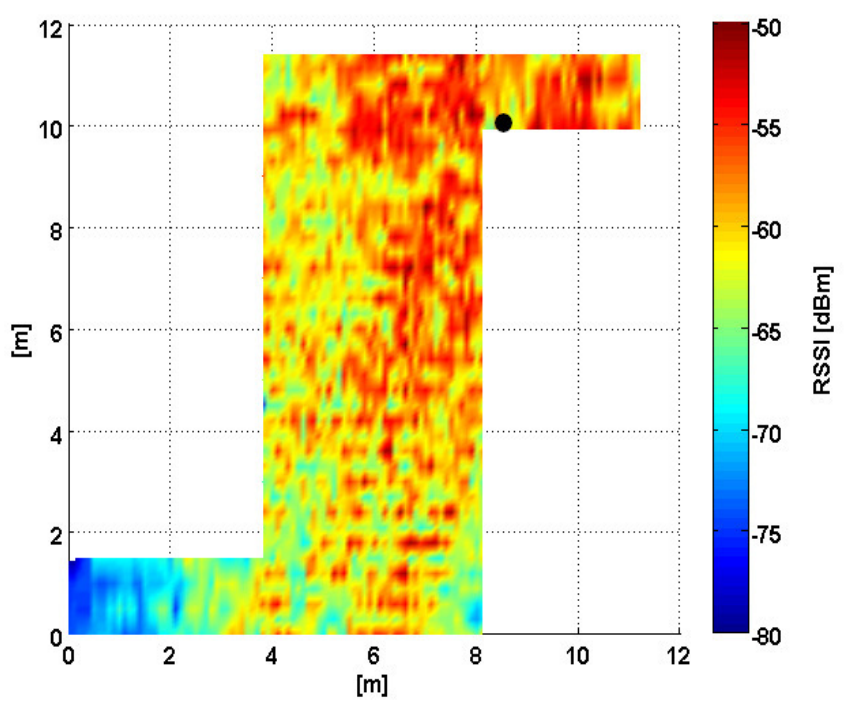

Fig 5. Signal strength map. The black point is AP.

realized with it. Based on the experiment with indoor RSSI measurements it can be stated that the Yrobot system enhanced by communication modules with wireless connectivity provides more functionality and allows development of many interesting applications such as precise localization of mobile systems using signal strength measurements.

In the next step we want to integrate the mobile platform into IoT environment using ThingWorx suite. We would like to expand the possibilities of the Yrobot platform further by developing of the additional modules for technologies such as RFID, NFC, Z-Wave and a chosen proprietary communication systems in the ISM bands (eg. RFM70).

\section{REFERENCES}

[1] J. Miček et. al., Sprievodca po svete Yrobota, University of Žilina, 2015.

[2] M. Kochláň, M. Hodoň, "Open hardware modular educational robotic platform - Yrobot", in Proc. 23rd International Conference on Robotics in Alpe-Adria-Danube Region (RAAD), Slovakia, 2014. http://dx.doi.org/10.1109/RAAD.2014.7002246

[3] J. Miček, O. Karpiš, "Audio communication subsystem of multirobotic system YROBOT", in Proc. 23rd International Conference on Robotics in Alpe-Adria-Danube Region (RAAD), Slovakia, 2014. http://dx.doi.org/10.1109/RAAD.2014.7002263

[4] J. Miček, O. Karpiš, M. Kochláň, "Audio-communication subsystem module for Yrobot - a modular educational robotic platform", in Proc. 6th European Embedded design in education and research (EDERC), Milano, 2014, pp. 60-64. http://dx.doi.org/10.1109/EDERC.2014.6924359 